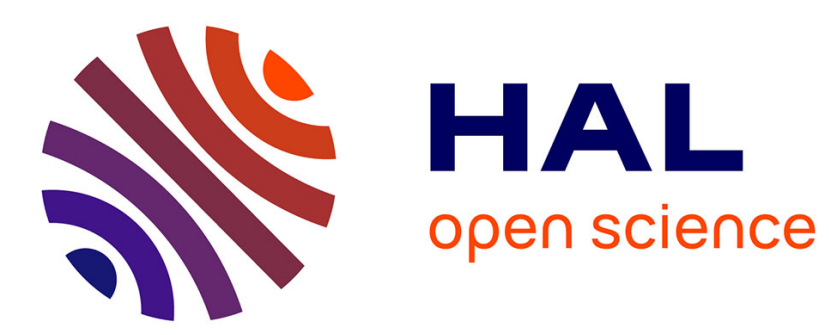

\title{
MAGNETIC F CENTER IN CHALCOGENIDE SPINEL TYPE FERROMAGNETIC DIVALENT IONIC CRYSTALS
}

\author{
H. Kamimura, Y. Natsume
}

\section{- To cite this version:}

H. Kamimura, Y. Natsume. MAGNETIC F CENTER IN CHALCOGENIDE SPINEL TYPE FERROMAGNETIC DIVALENT IONIC CRYSTALS. Journal de Physique Colloques, 1973, 34 (C9), pp.C9-281-C9-283. 10.1051/jphyscol:1973949 • jpa-00215424

HAL Id: jpa-00215424

https://hal.science/jpa-00215424

Submitted on 1 Jan 1973

HAL is a multi-disciplinary open access archive for the deposit and dissemination of scientific research documents, whether they are published or not. The documents may come from teaching and research institutions in France or abroad, or from public or private research centers.
L'archive ouverte pluridisciplinaire HAL, est destinée au dépôt et à la diffusion de documents scientifiques de niveau recherche, publiés ou non, émanant des établissements d'enseignement et de recherche français ou étrangers, des laboratoires publics ou privés. 


\title{
MAGNETIC F CENTER IN CHALCOGENIDE SPINEL TYPE FERROMAGNETIC DIVALENT IONIC CRYSTALS
}

\author{
H. KAMIMURA and Y. NATSUME \\ Department of Physics, Faculty of Science, University of Tokyo, Tokyo (Japan)
}

\begin{abstract}
Résumé. - Afin d'interpréter les caractéristiques du pic saillant de dédoublement dans le rouge observé dans $\mathrm{CdCr}_{2} \mathrm{~S}_{4}$, un modèle de centre $\mathrm{F}$ magnétique est proposé, constitué de deux électrons piégés dans une lacune anionique avec des spins parallèles. Il est montré par la méthode LCAO que l'état fondamental est ${ }^{3} \mathrm{E}$ au-dessous de $T_{\mathrm{c}}$ et que le pic est dû à une transition vers son état excité.
\end{abstract}

\begin{abstract}
Magnetic F center model consisting of two electrons trapped at an anion vacancy with their spins being parallel is proposed in connection with of the prominent red shift peak observed in $\mathrm{CdCr}_{2} \mathrm{~S}_{4}$. With LCAO method it is shown that its ground state is ${ }^{3} \mathrm{E}$ below $T_{\mathrm{c}}$ and the peak is due to transition to its excited state.
\end{abstract}

1. Introduction. - Recently much attention has been paid to optical properties and electronic structures of chalcogenide spinel type ferromagnetic ionic crystals. These crystals are classified into two types, Typed I or Type II, according as their energy gaps are smaller or larger than the strength of the cubic crystal field for the $\mathrm{Cr}^{3+}$ ions at the $\mathrm{B}$ sites. For example, $\mathrm{CdCr}_{2} \mathrm{Se}_{4}$ [1] and $\mathrm{HgCr}_{2} \mathrm{Se}_{4}$ [2] belong to the former while $\mathrm{CdCr}_{2} \mathrm{~S}_{4}$ [3] belong to the latter. In the Type II materials, their optical properties have a feature such that the crystal field transitions in the $\mathrm{Cr}^{3+}$ ions appear below the fundamental absorption band edge [4]. In $\mathrm{CdCr}_{2} \mathrm{~S}_{4}$, besides these crystal field transitions, the observation of a prominant red shift peak in the absorption band edge has been reported by Berger and Ekstrom [3], who have called this peak the C-peak.

In view of the fact that no theoretical attempt has been made to clarify the origin of this peak, we propose in this paper a possible model which explains the appearances of this peak. Before explaining our model, the observed features of this peak are firstly mentioned:

1) The C-peak appears below $T_{\mathrm{c}}$ and shifts to lower energy as temperature decreases [3].

2) The peak shows the magnetic circular dichroism. Namely, the peak appears only for the right circularly polarized light $(m=+1)$ in the Faraday configuration in which the light propagates along the magnetic field [3].

3) The large magnetic Kerr rotation is observed near the energy of the C-peak [5], [6].

4) The $f$-value of the peak is about $10^{-4}[3]$.

5) The peak is sensitive to doping and sample stoichiometry [3].

In order to explain these observed features, we propose a magnetic $F$ center model which consists of two electrons trapped at a vacancy of a $\mathrm{S}^{2-}$ ion with their spins being parallel. Namely, it is shown that the magnetic $\mathrm{F}$ center is possible to exist in the Type II ferromagnetic divalent ionic crystals and further that the $C$-peak is due to this $F$ center.

2. Electronic structure of magnetic $\mathbf{F}$ center. For the present purpose we calculate the one electron orbital energies of this center in the presence of the exchange interactions due to the $\mathrm{Cr}^{3+}$ spins in a crystal.

As shown in figure 1, the $S$ ion vacancy is surrounded by a $\mathrm{Cd}^{2+}$ ion and three $\mathrm{Cr}^{3+}$ ions and it has $C_{3 \mathrm{v}}$ symmetry. Since the trapped electrons are expected to be in the vicinity of the vacancy, it may be reasonable to approximate the one-electron wave functions of the trapped electrons by the linear combination of atomic orbitals of the surrounding $\mathrm{Cd}$ and three $\mathrm{Cr}$ ions (the LCAO MO method).

Constructing the LCAO MO orbitals of the trapped electrons with a $5 \mathrm{~s}$ orbital of the $\mathrm{Cd}^{2+}$ ion, $\varphi_{5 \mathrm{~s}}$, and the vacant $e_{g}$ orbitals of the three $\mathrm{Cr}^{3+}$ ions, $\chi_{u}^{2}, \chi_{u}^{3}$,

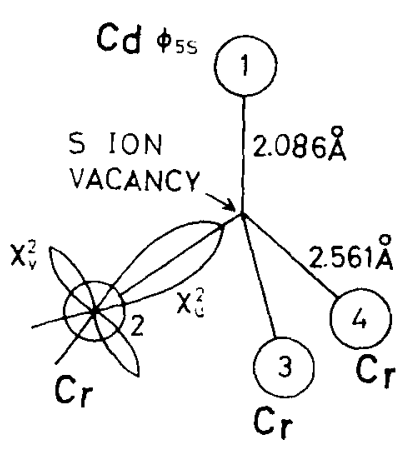

FIG. 1. - The system of a $S^{2-}$ ion vacancy. The angular behavior of the $e_{g}$ wave functions, $\chi_{u}$ and $\chi_{v}$, are also shown in the figure. 
$\chi_{u}^{4}, \chi_{v}^{2}, \chi_{v}^{3}, \chi_{v}^{4}$ as shown in figure 1, we can calculate the energy of each molecular orbital. The molecular orbitals are classified by the irreducible representations of the $C_{3 v}$ group and these are shown in table I. The calculated orbital energies are shown in figure 2. An orbital with the lowest energy is the non-degenerate $a_{1}$ orbital, and the first excited orbital is the doubly degenerate $e$ orbital, $e(v)$ and then $e(u), a_{2}(u)$ and antibonding $a_{1}(u)$ in order of increasing energy.

In order to accommodate two electrons in these orbitals, we must take into account first of all the

\section{TABLE I}

The classification of the molecular orbitals by the irreducible representations of the $C_{3 \mathrm{v}}$ group

$$
\begin{aligned}
& \text { Represen- } \\
& \text { tations Cadmium Chromium } \\
& a_{1}(u) \quad \frac{1}{\sqrt{\left(1+\rho^{2}\right)}}\left\{\rho \varphi_{5 s}+\frac{1}{\sqrt{3}}\left(\chi_{u}^{2}+\chi_{u}^{3}+\chi_{u}^{4}\right)\right\} \\
& a_{1}(u)^{*} \quad \frac{1}{\sqrt{\left(1+\rho^{2}\right)}}\left\{\varphi_{5 \mathrm{~s}}-\frac{\rho}{\sqrt{3}}\left(\chi_{u}^{2}+\chi_{u}^{3}+\chi_{u}^{4}\right)\right\} \\
& a_{2}(v) \quad \frac{1}{\sqrt{3}}\left(\chi_{v}^{2}+\chi_{v}^{3}+\chi_{v}^{4}\right) \\
& e(u) \quad \frac{1}{\sqrt{2}}\left(\chi_{n}^{2}-\chi_{u}^{3}\right) \\
& \frac{1}{\sqrt{6}}\left(\chi_{u}^{2}+\chi_{u}^{3}-2 \chi_{u}^{4}\right) \\
& e(v) \\
& \frac{1}{\sqrt{2}}\left(\chi_{v}^{2}-\chi_{v}^{3}\right) \\
& \frac{1}{\sqrt{6}}\left(\chi_{v}^{2}+\chi_{v}^{3}-2 \gamma_{v}^{4}\right)
\end{aligned}
$$

\section{The Spin-Orbit splitting}

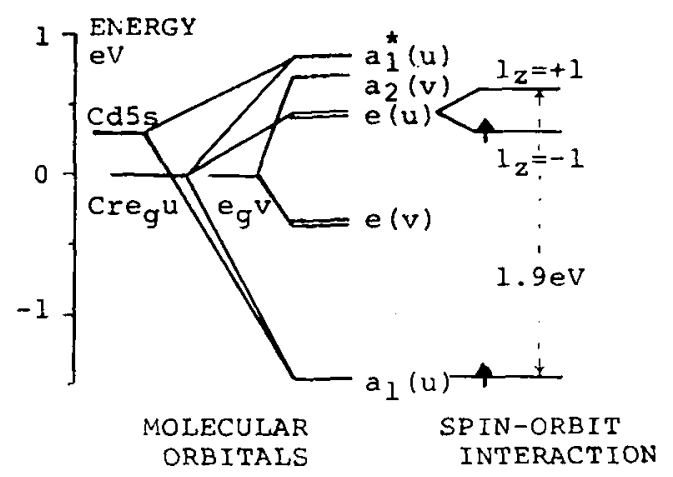

FIG. 2. - The energy level diagram of the magnetic $F$ center and its optical transition. exchange interaction between the spins of the trapped electrons and of the surrounding three $\mathrm{Cr}^{3+}$ ions which makes the spins of the trapped electrons parallel, and then the coulomb and exchange interaction between the trapped electrons. We can express the former exchange interaction in the following approximate form, adopting the molecular field approximation for the exchange interaction between the localized spins of the $\mathrm{Cr}^{3+}$ ions in a whole crystal:

$H_{\mathrm{eI}}=-\sum_{i=1,2} \sum_{j=\text { Crions }} I\left(r-R_{j}\right) s_{i z}<S_{j z}>_{T}$

where the summation over the $\mathrm{Cr}$ ions is taken only over the surrounding three $\mathrm{Cr}$ ions numbered by 2,3 , 4 (see Fig. 1), and $s_{i z}$ and $S_{j z}$ are the $z$-components of the spins of the $i$-th trapped electron $(i=1$ and 2) and the $j$-th $\mathrm{Cr}$ ion, respectively.

Further $\langle A\rangle_{T}$, represents the thermal average of $A$ at temperature $T$, and the $z$-axis is taken along the direction of the total magnetization due to the $\mathrm{Cr}^{3+}$ spins in a whole crystal. For the exchange integral $I\left(r-R_{j}\right)$ in $(1)$, we assume

$$
I\left(r-R_{j}\right)=I \delta\left(r-R_{j}\right) .
$$

Namely, the trapped electrons interact with the localized spins of the surrounding $\mathrm{Cr}^{3+}$ ions only when they come to the $\mathrm{Cr}^{3+}$ sites and their spins become parallel to the $\mathrm{Cr}^{3+}$ spins. Thus for the value of $I$ we take the intra-atomic exchange integral between the $e_{g}$ and $t_{2 g}$ orbitals in a $\mathrm{Cr}^{2+}$ ion with $S=2$ at a site with cubic symmetry.

It is clear from the above fact that two trapped electrons with parallel spins must occupy different molecular orbitals because the lowest $a_{1}(u)$ orbital is non-degenerate.

Since the coulomb interaction between two electrons at the same ion is very large such as the order of $10 \mathrm{eV}$, an electron configuration in which two electrons do not meet at the same ion has lower energy. Thus we find that the electron configuration in which one electron occupies the $a_{1}(u)$ orbital and the other occupies the higher $e(u)$ orbital with their spins being parallel rather than lower $e(v)$ orbital has the lowest energy. As a result, the ground state of the $\mathrm{F}$ center is ${ }^{3} \mathrm{E}$ below $T_{\mathrm{c}}$ with the electron configuration $\left(a_{1}(u)\right)^{1}$ $(e(u))^{1}$ with the spin triplet. Because of this we call this $\mathrm{F}$ center as magnetic.

On the basis of this energy diagram, the C-peak can be assigned to the transition from $a_{1}$ to $e$ orbital, by choosing the ratio of the mixing of the $\mathrm{Cd} 5 \mathrm{~s}$ orbital in the $a_{1}$ orbital, $\rho$, so as to fit the energy separation between $a_{1}$ and $e$ orbitals to the peak position of the $\mathrm{C}$-peak $(1.9 \mathrm{eV})$. The value of $\rho$ thus determined is 0.55 .

3. Optical properties of the magnetic $F$ center. Now we will show that the observed features of the C-peak mentioned before can be explained by this assignment. 
3.1 Since the $a_{1}$ orbital is composed of both the $\mathrm{Cd} 5 \mathrm{~s}$ and $\mathrm{Cr} e_{g}$ orbitals while the $e$ orbitals is formed from only the $\mathrm{Cr} e_{g}$ orbitals, the decrease in the energy of the $a_{1}$ orbital by $H_{\mathrm{e} I}$ is smaller than that in the $e$ orbital. Thus the transition energy from $a_{1}$ to $e$ orbital below $T_{\mathfrak{c}}$ is smaller than that above $T_{0}, E_{0}$.

Namely, the transition energy below $T_{c}$ is expressed as

$$
\begin{aligned}
E\{e(u)\}-E & \left\{a_{1}(u)\right\}= \\
& =E_{0}-\frac{\rho^{2}}{1+\rho^{2}} \cdot I L S_{z}>_{T} .
\end{aligned}
$$

Because of $\left\langle S_{j z}\right\rangle_{T}$ in (3), this energy becomes smaller as temperature decreases from $T_{\mathrm{c}}$, and thus the C-peak shows the red shift. The value of this shift at $T=0$ is estimated to be about $0.29 \mathrm{eV}$ and this is in fair agreement with the observed values of 0.12 to $0.15 \mathrm{eV}$ [3].

3.2 The ground state of the magnetic $F$ center is orbitally doubly degenerate. Therefore, the spin-orbit interaction plays an important role. Namely, because of the spin-orbit interaction the ${ }^{3} \mathrm{E}$ state is split into two. These states are classified according as the $z$ component of the total orbital angular momentum $L_{z}$ takes a value of +1 or -1 , and the state of $L_{z}=-1$ has the lower energy. Since the $C$-peak corresponds to the transition from $a_{1}$ to the $l_{z}=+1$ component of the $e$ orbital as shown in the extreme right of figure 2 , the value of $L_{z}$ in the excited state is zero. Thus the $C$-peak appears only in the right circularly polarized light, corresponding to the change of $L_{z}$ from -1 to 0 .

3.3 In connection with the above polarization behavior the magnetic Kerr rotation is calculated as a function of the frequency in the region of the C-peak position. It is shown that the real and imaginary parts of the off-diagonal element of the dielectric tensor are of dispersive and dissipative types, respectively, for the C-peak. This behavior is in good agreement with experiments [6].

3.4 Concerning the $f$ value of the $\mathrm{C}$-peak, it is estimated to be about $10^{-3}$ to $10^{-4}$ in agreement with the observed value. This is easily understandable from the fact that the transition from $a_{1}$ to $e$ orbital is of the character of the $d-d$ transition in a $\mathrm{Cr}^{2+}$ ion.

3. 5 This transition, of course, is sensitive to doping and sample stoichiometry.

We have seen above that the magnetic $F$ center model can explain the appearance of the C-peak below $T_{c}$ and its prominent features successfully. Thus we can conclude that the triplet $F$ center may exist in a magnetic divalent ionic crystal.

4. Conclusion. - We have shown that the magnetic $F$ center may exist in ferromagnetic divalent ionic crystals such as $\mathrm{CdCr}_{2} \mathrm{~S}_{4}$ and that the optical transition between the electronic level in the $F$ center is responsible for the appearance of the $C$-peak in $\mathrm{CdCr}_{2} \mathrm{~S}_{4}$. The magnetic $\mathrm{F}$ center is characteristic of magnetic divalent ionic crystals, because in nonmagnetic divalent ionic crystals such as $\mathrm{MgO}$ the ground state of the F center is singlet [7]. However, it should be remarked that, above $T_{c}$ the exchange interaction $H_{\mathrm{cl}}$ disappears and thus the ground state of the $\mathrm{F}$ center in ferromagnetic crystals may become ${ }^{1} \mathrm{~A}$ in which two electrons occupy the lowest $a_{1}(u)$ orbital with their spins being antiparallel [8]

\section{References}

[1] Harbeke, G. and Lehmann, H. W., Sol. State Commun. 8 (1970) 1281.

[2] Lehmann, H. W. and Emmenegger, F. P., Sol. State Commuli. 7 (1969) 965.

[3] Berger, S. B. and Eкstrom, L., Phys. Rev. Lett. 23 (1969) 1499 .

[4] Natsume, Y., to be published.

[5] WIтTeKock, S, and Bongers, P. F., IBM Magnetic Semiconductor Conference, 1969.
[6] Wittekock, S. and Rinzema, G., Phys. Stat. Sol. (b) 44 (1971) 849

[7] Henderson, B., Stokowski, S. E. and Ensign, T. C., Phys. Rey. 183 (1969) 826.

[8] Near the Curie temperature we have to consider the effects of the short range order which have not been included in the present treatment. These effects may allow the existence of the triplet state of the $F$ center even above the Curie temperature. 